\title{
Health Resource Allocation in Western China from 2014 to 2018
}

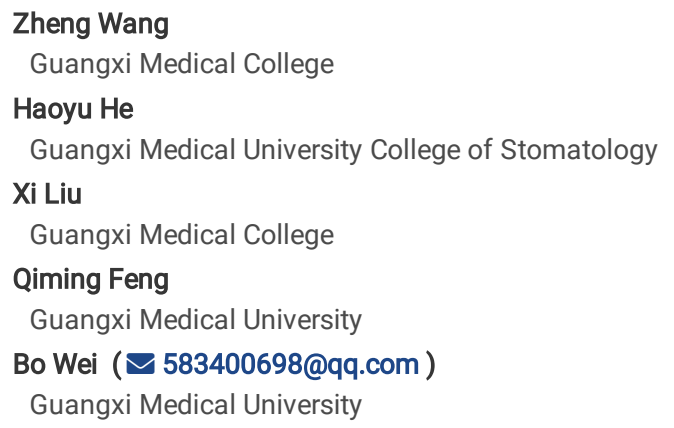

\section{Research}

Keywords: Western China, Health resources allocation, Gini coefficient

Posted Date: August 13th, 2021

DOI: https://doi.org/10.21203/rs.3.rs-792643/v1

License: () (7) This work is licensed under a Creative Commons Attribution 4.0 International License. Read Full License 


\section{Abstract}

Background: Health equity has persistently been a global concern. As a basic material guarantee to ensure health equity, how to allocate the health resources in a fair and reasonable way has always been one of the research hotspots. The coronavirus 2019 (COVID-19) pandemic has also prompted a rethinking of the topic. Based on the previous research, western China is a relatively backward region, coupled with climate, geography, and other factors, which leading the inconvenient transportation and difficult resource allocation. However, the fairness of health resource allocation in western China has received relatively little attention.

Methods: Lorentz curve, Gini coefficient and Theil index were used to analyse the health resources allocation in the western China. The indexes include number of beds, medical (assistant) practitioners, registered nurses from 2014 to 2018 through population and geography dimensions.

Results: The total health resources shows an increasing trend from 2014 to 2018; The Lorentz curve in the population dimension had a smaller curvature than in the geography dimension. The Gini coefficients for health resources in the population dimension were ranged from 0.057 to 0.129 , and in geography dimension the Gini coefficients ranged between 0.605 and 0.647 . This shows that the distribution of Health resources is fair basing on population dimension. Furthermore, in two dimensions, the intra-group contribution rate of the Theil index was higher than in the inter-group, and the allocation of practicing (assistant) practitioners and registered nurses show a significant inequity in intra-group. This result indicated that the inequity of health resources allocation were mainly came from intra-group, namely the provinces (autonomous regions and municipalities) in western China.

Conclusions: In recent years, China's various measures have improved the total amount of health resources and its equity of health resources in western China. However, the fairness of the health resources allocation in western China were remind poor. Although the fairness of human resources allocation has been alleviated in population dimension, the inequity of human resources distribution in provinces (autonomous regions and municipalities) are still obvious. In addition, the accessibility of health resources in western China must be further improved.

\section{Background}

The concept of health equity was first proposed in the United Kingdom, where the government's Health Inequalities Panel described the social health disparities and attributed the disparities to the difference of socioeconomic environment. This concept has attracted the attention of the global health community[1]. Health equity includes equity of health financing, equity of health service utilisation and equity of health results. -Health resource distribution is one of the contents of equity of health service utilisation, which significantly impacts health equity.

The distribution of health resources refers to how the government or the market distributes health resources fairly and efficiently amongst different areas, regions, departments, projects, and groups, to maximise the social and economic benefits of health resources. The distribution of health resources is also recognised as the basis for the sustainable development of health undertakings, it also plays an important role in ensuring the performance of the health system to reaches the standard. The fairness of the health resources allocation, as the premise of the fairness of the utilisation of health services, is causally related to the health right of the people [2-4]. Health equity is incredibly significant that it has attracted considerable worldwide attention. As the material basis for maintaining people's health, optimising health resources allocation has been a hot research topic in recent years[5]. During the outbreak of the COVID-19 pandemic, the scholars started further exploring how to rationally allocate health resources to ensure that residents receive them timely and effectively [6].

China also attaches great importance to fair allocation of resources. In the "Healthy China 2030" plan released in 2016, it is clearly proposed to "gradually reduce the differences in basic health services and health levels between urban and rural areas, regions and groups of people, achieve universal health coverage and promote social health equity". To analyse the equity of health resources allocation in China, many scholars have conducted studies. For instance, Wang Shuping et al. analysed the trend of equity of primary health resources allocation in Liaoning province[7]. Xu Kang et al. analysed the distribution trend of primary health care workforce in Jiangsu province[8]. Ren Zhenghong et al. analysed the distribution of human resources in Obstetrics and gynaecology in China[9]. Sun Jian et al. analysed the equity and efficiency of health resource allocation and health service utilization in China[10]. These studies have provided strong support to the policy maker, however, there were few studies focus on the western region.

Western China is a developing region in China. Its economic and medical developments lags behind other areas in China. Western of China includes 12 provinces (Autonomous Regions and Municipalities), namely Shaanxi, Sichuan, Yunnan, Guizhou, Gansu, Qinghai, Guangxi Zhuang Autonomous Region, Ningxia Hui Autonomous Region, Tibet Autonomous Region, Xinjiang Uygur Autonomous Region, Inner Mongolia Autonomous Region and Chongqing Municipality. By the end of 2018, western of China accounted for 70.6 percent of the country's total area, and its population was 379.5587 million, accounting for 27.2 percent of the total population, it is an extremely significant part of China. Western China is a vast territory, with a relatively backward economy than other areas, and it is mostly mountainous, which hinders transportation. These factors increase the difficulty of health resources distribution and affect the health level of the people in western China. Based on the relevant research, the equity of health resource allocation in the western China was lower than that in other developed regions, which requires greater attention [11-13]. Nonetheless, there exist few studies that focused on the equity of health resource allocation in western China and the factors that may affect the distribution equity in western China. Therefore, the allocation equity of health resource in western China must be studied, and the influencing factors must be identified. This study aims to analyse the distribution equity of health resources in western China and put forward corresponding countermeasures and suggestions on the basis of the analysis results to further promote the equity of health resource allocation.

\section{Data Sources And Methods}

\section{Data sources}


Public data were collected by searching the National 2015-2019 Statistical Yearbook [14], the data released by local health commissions during 2015-2019, and Statistical Yearbook of the Western Provinces (Autonomous Regions and Municipalities) of China 2015-2019 [15-26]. According to 'The 13th Five-Year Plan for Health and Wellness', the resources in medical and health service system includes number of beds in health care facilities, medical (assistant) physician, registered nurses, general practitioners, and proportion of beds in socially run hospitals in total number of hospital beds. Considering of the data accessibility, the data collected this time were mainly include number of beds, medical (assistant) physicians, registered nurses, population, and area in western China from 2014 to 2018.

\section{Functional regional division}

The 12 provinces were divided into two areas: Ethnic minority area and non-ethnic minority areas. Ethnic minority area includes the Ningxia Hui Autonomous Region, the Tibet Autonomous Region, the Xinjiang Uygur Autonomous Region and the Inner Mongolia Autonomous Region, the rest of the provinces were divided into non-minority areas.

\section{Measurements of inequity}

Lorenz curve, Gini coefficient and Theil index have their own advantages and disadvantages in analysing health resources allocation. Comprehensive use of these analytical methods can objectively analyse the fairness of health resources allocation and determine the main factors affecting the inequity of resources allocation. Hence, the three measurements were used to analyse the equity of distribution in western of China in population dimension and geographical dimensions.

\section{Lorentz curve}

At the early period, Lorenz Curve was mainly used to evaluate the equity of resource or income distribution and measure the equity difference. Now, it is commonly used to measure the equity of health care resources[8]. Its drawing method is classified into people's income or resources according to different groups or regions, builds a coordinate system with the cumulative percentage of population (population dimension) or land area (geographical dimension) and the cumulative percentage of various medical resources as coordinates. Then, connected to the zero point and each coordinate point curve drawing to draw an absolute fairness line. The fairness in Lorentz is judged on the basis of its distance from the absolute fairness line. The closer it is to the absolute fairness line, the fairer it is, and the worse it is[27].

\section{Gini coefficient}

Lorentz curve can evaluate the equity of resources. However, it cannot quantify the specific difference[28]. Therefore, Gini coefficient must be used for further accurate analysis. Gini coefficient is calculated as follows[29]:

$$
G=\frac{1}{2 n^{2} \mu} \sum_{i-1}^{n} \sum_{j-1}^{n}\left|y_{i}-y_{j}\right| \quad \text { (formula 1) }
$$

Where, $G$ stands for Gini coefficient, $N$ for group number, $\mu$ for resident income, $Y_{i}$ and $Y_{j}$ for per capita income of group I and J[30].

Gini coefficient value range is $[0,1]$, the closer it gets to 0 , the fairer it is. Meanwhile, the closer it is to 1 , the less fair it is. It is generally believed that: Gini coefficient less than 0.3 means the resources allocation is in a best fair state; $0.3-0.4$ is the normal state; a level of alert above 0.4 ; above 0.6 is in a dangerous state of high unfairness[31, 32].

\section{Theil index}

Theil index is the major instrument to measure fairness, it can directly reflect the influence of different regions to resource allocation. Its value range is [0,1],the lower the value of Theil index, the better the balance of social resource allocation in this region [33,34]. Given that the Theil index can divide the overall variance into the differences between groups, hence, Theil index was used to analyse the health resource allocation, the equity of health resource allocation between and within different regions within the region can also be measured[35].

The calculation formula of Theil index is as follows:

$$
\begin{array}{ll}
\text { Theil }-l=\sum_{i-1}^{n} P_{n} \log _{\frac{P_{n}}{Y n}} & \text { (formula 2) } \\
T_{\text {inter }}=\sum_{g-1}^{k} P_{j} \log _{\frac{P_{j}}{Y j}} & \text { (formula 3) } \\
T_{\text {intra }}=\sum_{g-1}^{k} P_{j} T_{j} & \text { (formula 4) } \\
\text { Theil }=T_{\text {inter }}+T_{\text {intra }} & \text { (formula 5) }
\end{array}
$$


Where, $P_{n}$ refers to the proportion of the population of each province in the total population; $P_{j}$ is the proportion of the population of each region in the total population; Each $Y_{n}$ is the proportion of resources of each province in the total number of resources on this dimension. $Y_{j}$ shows the proportion of resources of each region in the total number of resources[36, 37]

\section{Results}

\section{Basic situation of health resources allocation in the western China from 2014 to 2018}

Tables 1-3 exhibits the distribution of health resources in 12 provinces (autonomous regions and municipality) in western China from 2014 to 2018 . The three indicators showed an upward trend. Based on the distribution of health resources in 2018, Xinjiang had the most beds per 1,000 people, 7.19, Sichuan (7.18) and Chongqing (7.10) followed,whereas Tibet had the least number of beds per thousand people (4.88), followed by Guangxi (5.20) and Ningxia (5.96). Inner Mongolia has the highest number of medical (assistant) partitioner per 1,000 people (2.90), followed by Ningxia (2.82) and Qinghai (2.68), whereas Yunnan has the lowest number, 2.06, followed by Guangxi (2.15) and Guizhou (2.26). Shaanxi had the most registered nurses per 1,000 people (3.57), followed by Ningxia (3.38) and Chongqing (3.07) whereas Xizang had the least (1.62),followed by Gansu (2.44) and Yunnan (2.83).

Table 1

The distribution of beds per 1,000 population in the western China in 2014-2018

\begin{tabular}{|llllll|}
\hline region & $\mathbf{2 0 1 4}$ & $\mathbf{2 0 1 5}$ & $\mathbf{2 0 1 6}$ & $\mathbf{2 0 1 7}$ & $\mathbf{2 0 1 8}$ \\
\hline Guangxi & 4.42 & 4.47 & 4.46 & 4.94 & 5.20 \\
\hline Shaanxi & 5.28 & 5.59 & 5.91 & 6.29 & 6.57 \\
\hline Gansu & 4.72 & 4.91 & 5.15 & 5.58 & 6.17 \\
\hline Qinghai & 5.66 & 5.87 & 5.86 & 6.41 & 6.49 \\
\hline Ningxia & 4.91 & 5.06 & 5.38 & 5.84 & 5.96 \\
\hline Xinjiang & 6.22 & 6.37 & 6.54 & 6.85 & 7.19 \\
\hline Sichuan & 5.65 & 5.96 & 6.28 & 6.79 & 7.18 \\
\hline Chongqing & 5.37 & 5.85 & 6.26 & 6.71 & 7.10 \\
\hline Yunnan & 4.77 & 5.01 & 5.31 & 5.72 & 6.03 \\
\hline Guizhou & 5.19 & 5.57 & 5.92 & 6.51 & 6.82 \\
\hline Inner Mongolia & 5.15 & 5.33 & 5.53 & 5.94 & 6.27 \\
\hline Tibet & 3.75 & 4.33 & 4.37 & 4.78 & 4.88 \\
\hline
\end{tabular}

Table 2

Distribution of medical staff per 1,000 population in westem China from 2014 to 2018

\begin{tabular}{llllllllll} 
& 2014 & & 2015 & & 2016 & & 2017 & 2018 \\
region & physician & nurse & physician & nurse & physician & nurse & physician & nurse & physician \\
\hline Guangurse
\end{tabular}


Table 3

Gini coefficients of health resources based on different dimensions in western China from 2014 to 2018

\begin{tabular}{|lllllll|}
\hline \multicolumn{3}{|l}{ Population dimension } & \multicolumn{4}{c|}{ Geographical dimension } \\
\hline Year & beds & practitioner & nurses & beds & practitioner & nurses \\
\hline 2014 & 0.0583 & 0.0785 & 0.0719 & 0.6275 & 0.6151 & 0.6359 \\
\hline 2015 & 0.0577 & 0.0694 & 0.0644 & 0.6300 & 0.6136 & 0.6388 \\
\hline $\mathbf{2 0 1 6}$ & 0.0584 & 0.0601 & 0.0611 & 0.6338 & 0.6158 & 0.6421 \\
\hline $\mathbf{2 0 1 7}$ & 0.0580 & 0.0578 & 0.0438 & 0.6344 & 0.6149 & 0.6433 \\
\hline $\mathbf{2 0 1 8}$ & 0.0574 & 0.0525 & 0.0462 & 0.6343 & 0.6200 & 0.6469 \\
\hline
\end{tabular}

\section{Lorentz curve of health resources in western China from 2014 to 2018}

Taking the cumulative population ratio as the X-axis and the cumulative percentage of health resources as the Y-axis, Lorentz curve under the population dimension was plotted, and the result was shown in Fig. 1. Similarly, the Lorentz curve under the geographical dimension was drawn with the geographical area as the $\mathrm{X}$-axis and the cumulative percentage of health resources as the $\mathrm{Y}$-axis. The results were shown in Figure. 2. Compare Lorentz curves in two dimensions, the curve in the geographical dimension was significantly distant from the absolute fairness line than in the population dimension, which indicates that the allocation of health resources in population dimension is better than that in geographical dimension. Moreover, the bending degree of Lorentz curves of the three analysis indicators in the population dimension is similar, indicating that the difference in fairness degree is small. The Lorentz curves of the three analysis indicators in the geographical dimension shows similar results.

\section{Gini coefficient of health resources in western China from 2014 to 2018}

Gini coefficients were calculated respectively according to the Lorenz curve drawing results of population and geographical dimensions, as shown in Table 3. In 2014, the three analysis indicators of health resources in the population dimension are between 0.058 and 0.078 , less than 0.2 , which indicates the best equity state. The Gini coefficient of the three health resources analysis indicators in the geographical dimension is between 0.615 and 0.636 , higher than 0.6 , showing a highly unfair state.

In 2018 , the three analysis indicators of health resources in the population dimension are between 0.046 and 0.057 , less than 0.2 , which indicates the best fairness. The Gini coefficient of the three health resources analysis indicators in the geographical dimension is between 0.620 and 0.647 , higher than 0.6 , showing a highly unfair state. Comparing the Gini coefficient in 2014 and 2018 , the equity of health resources allocation in population dimension is better than that in geographical dimension, furthermore, the three analysis indicators have similar values in each dimension, indicating that the fairness difference in each dimension is small. This result verifies the result of Lorentz curve drawing.

In addition, Fig. 3 exhibits that the Gini coefficient in the population dimension presents a downward trend, which indicated that that equity has improved. Meanwhile the Gini coefficient on the basis of the geographical area, presents an overall gradual upward trend, as shown in the Fig. 4.

\section{Theil index of health resources in western China}

Based on the Theil index analysis of different dimensions shown in Table 4, Theil index in population dimension is lower than that in geographical dimension, which indicates that the allocation of health resources in population dimension is better. Moreover, it can be seen from the analysis results of Theil index contribution rate of health resource allocation from the two dimensions in Table 5,the Theil index in the two dimensions shows a status of intra-group higher than inter-group. From the perspective of population dimension, the three indexes were all showed that the intra-group contribution rate greater than the intergroup contribution rate, and the contribution rate were all reached more than $70 \%$. Among them, the contribution rate of practicing (assistant) physicians and registered nurses in population dimension was the most obvious. The above results indicate that the inequality of resource allocation mainly comes from intra-group, namely inside the provinces (autonomous regions, municipalities), and the inequity allocation of human resources (practicing (assistant) doctors and registered nurses) is obvious. The contribution rate in geographical dimension shown a similar tendency, and the rate has reached above $60 \%$. 
Table 4

Theil index of health resources based on two dimensions in western China from 2014-2018

\begin{tabular}{|c|c|c|c|c|c|c|c|c|c|c|}
\hline \multirow[t]{2}{*}{ Dimensions } & \multirow[t]{2}{*}{ Year } & \multicolumn{3}{|l|}{ Beds } & \multicolumn{3}{|c|}{ Practitioner } & \multicolumn{3}{|c|}{ Nurses } \\
\hline & & $\mathrm{T}$ & $\mathrm{T}_{\text {intra }}$ & $\mathrm{T}_{\text {inter }}$ & $\mathrm{T}$ & $\mathrm{T}_{\text {intra }}$ & $T_{\text {inter }}$ & $\mathrm{T}$ & $T_{\text {intra }}$ & $\mathrm{T}_{\text {inter }}$ \\
\hline \multirow[t]{5}{*}{ Population } & 2014 & 0.002 & 0.002 & $0.000^{*}$ & 0.004 & 0.004 & 0.000 & 0.004 & 0.004 & 0.000 \\
\hline & 2015 & 0.002 & 0.002 & 0.000 & 0.003 & 0.003 & 0.000 & 0.003 & 0.003 & 0.000 \\
\hline & 2016 & 0.002 & 0.002 & 0.000 & 0.003 & 0.002 & 0.000 & 0.003 & 0.003 & 0.000 \\
\hline & 2017 & 0.002 & 0.002 & 0.001 & 0.002 & 0.002 & 0.000 & 0.002 & 0.002 & 0.000 \\
\hline & 2018 & 0.002 & 0.002 & 0.001 & 0.002 & 0.002 & 0.000 & 0.002 & 0.002 & 0.000 \\
\hline \multirow[t]{5}{*}{ Geographical } & 2014 & 0.318 & 0.200 & 0.117 & 0.300 & 0.204 & 0.096 & 0.228 & 0.097 & 0.228 \\
\hline & 2015 & 0.321 & 0.202 & 0.119 & 0.299 & 0.204 & 0.095 & 0.230 & 0.099 & 0.230 \\
\hline & 2016 & 0.326 & 0.205 & 0.121 & 0.301 & 0.206 & 0.096 & 0.231 & 0.102 & 0.231 \\
\hline & 2017 & 0.326 & 0.204 & 0.122 & 0.300 & 0.202 & 0.098 & 0.227 & 0.108 & 0.227 \\
\hline & 2018 & 0.326 & 0.203 & 0.123 & 0.306 & 0.205 & 0.101 & 0.227 & 0.111 & 0.227 \\
\hline
\end{tabular}

Table 5

Theil index of health resources based on two dimensions in western China from 2014-2018

\begin{tabular}{|c|c|c|c|c|c|c|c|}
\hline \multirow[t]{2}{*}{ Dimensions } & \multirow[t]{2}{*}{ Year } & \multicolumn{2}{|l|}{ Beds } & \multicolumn{2}{|c|}{ Practitioner } & \multicolumn{2}{|l|}{ Nurses } \\
\hline & & $\mathrm{T}_{\text {intra }}$ & $\mathrm{T}_{\text {inter }}$ & $\mathrm{T}_{\text {intra }}$ & $\mathrm{T}_{\text {inter }}$ & $\mathrm{T}_{\text {intra }}$ & $\mathrm{T}_{\text {inter }}$ \\
\hline \multirow[t]{5}{*}{ Population } & 2014 & $8.00 \%$ & $92.00 \%$ & $10.31 \%$ & $89.69 \%$ & $8.82 \%$ & $91.18 \%$ \\
\hline & 2015 & $12.53 \%$ & $87.47 \%$ & $14.15 \%$ & $85.85 \%$ & $6.90 \%$ & $93.10 \%$ \\
\hline & 2016 & $18.68 \%$ & $81.32 \%$ & $15.69 \%$ & $84.31 \%$ & $2.96 \%$ & $97.04 \%$ \\
\hline & 2017 & $23.72 \%$ & $76.28 \%$ & $10.68 \%$ & $89.32 \%$ & $0.12 \%$ & $99.88 \%$ \\
\hline & 2018 & $26.75 \%$ & $73.25 \%$ & $4.18 \%$ & $95.82 \%$ & $2.57 \%$ & $97.43 \%$ \\
\hline \multirow[t]{5}{*}{ Geographical } & 2014 & $36.95 \%$ & $63.05 \%$ & $32.04 \%$ & $67.96 \%$ & $29.87 \%$ & $70.13 \%$ \\
\hline & 2015 & $36.99 \%$ & $63.01 \%$ & $31.80 \%$ & $68.20 \%$ & $30.01 \%$ & $69.99 \%$ \\
\hline & 2016 & $37.21 \%$ & $62.79 \%$ & $31.77 \%$ & $68.23 \%$ & $30.55 \%$ & $69.45 \%$ \\
\hline & 2017 & $37.55 \%$ & $62.45 \%$ & $32.57 \%$ & $67.43 \%$ & $32.26 \%$ & $67.74 \%$ \\
\hline & 2018 & $37.71 \%$ & $62.29 \%$ & $33.09 \%$ & $66.91 \%$ & $32.85 \%$ & $67.15 \%$ \\
\hline
\end{tabular}

\section{Discussion}

This study analyses the equity of distribution in western China from 2014-2018, supplemented the analysis of health resource allocation in western China and provides reference for policy makers in the Chinese government.

On the basis of the analysis, we found that firstly, the overall health resources showed an increasing trend, however, the total health resources must be further improved. From 2014 to 2018 in western China, every 1000 people were allocated medical (assistant) practitioners, registered nurses, and number of beds which showed a trend of steady growth, indicating that Chinese government obtained some achievements in increasing health resources in western China, and residents' medical needs had been better meet. which is consistent with the research results of Ma Lu et al. [38]. However, according to 'China 13th FiveYear Health and wellness Plan', there should be six beds in medical institutions, 2.5 medical (assistant) practitioners and 3.14 registered nurses ever per 1,000 people [39]. As can be seen from Table 1 and table 2, in 2018, except for Guangxi and Tibet, the number of medical beds for every per 1,000 people basically reached the expectation of the plan. However, there were only 5 provinces (autonomous regions and municipalities) - Shanxi, Qinghai, Ningxia, Xinjiang and Inner Mongolia - have reached the expectation of the number of medical (assistant) practitioners for per 1,000 people in the 'China 13th Five-Year Health and wellness Plan'. The situation of registered nurses was more serious, only Shaanxi and Ningxia provinces had over 3.16 registered nurses for every per 1,000 people, meeting expectations. It can be seen that there is still a gap between the total amount of health resources in western China and the "13th Five-Year Plan", especially human resources. Moreover, in 2018 , there were 6 beds, 2.59 medical (assistant) practitioners and 2.94 registered nurses for every 1,000 people in the National Health Service[40].Comparing the relevant data of western China exhibited that the health resources for every 1000 people in western China were generally lower than the national average. By comparison, Zhejiang province, which located in the more developed eastern region of China, has 6.65 beds, 3.33 medical (assistant) practitioners and 3.51 registered nurses per 1,000 people in 2018[41]. Shandong province had 6 beds, 2.89 practicing 
(assistant) practitioners and 3.21 registered nurses per 1,000 people in 2018[42]. Hunan province, which located in central China, had 6.6 beds, 2.62 medical (assistant) practitioners and 2.67 registered nurses per 1,000 people [43]. Hubei province, which also located in central China, had 6.37 beds, 2.57 medical (assistant) physicians, and 3.23 registered nurses per 1,000 people[44]. The four representative provinces in eastern and central China is basically at or above the average level of China, The compared results shows that the total health resources in western China still must be improved. This may be related to the underdeveloped economy in western China and the tendency of government health resource allocation. The government is the main force to increase the total amount of medical resources. Therefore, the government must strengthen its functions in health resources allocation, enhance the feasibility of policies, strengthen relevant safeguard measures, and ensure the implementation of relevant policies to improve the total amount of health resources in the underdeveloped areas in western China, and further realize the goal of 'health equity for all' which emphasized in the '13th Five-Year Plan' and the 'Outline of healthy China 2030 Plan'[45].

Secondly, the geographical dimension of health resource allocation equity must be improved. Based on the analysis results of Lorenz curve, the curve in population dimension were nearer to the curve of absolute equality than that in the geographical dimension. Gini coefficient and Theil index were also lower in population dimension than that in geographic dimension. These results implied that the health resource allocation had more equity in the population dimension than that in the geographic dimension, and the inequity distribution of health resources in western China still shown an increased tendency. This result is basically consistent with the related studies[46,47]. The reason may be is that currently, China mainly chooses to allocation the health resources on the basis of the population density [48-50]. Hence, the distribution of health resources in the geographical dimension was more unequal also extending in other provinces and cities[51,52]. WHO recommends that everyone have access to affordable, quality health care services. However, this allocation standard can easily lead to problems such as small service radius, low service accessibility and low resource utilization, which may affect the population's easier access to health resources, and inconsistent with the GOAL of "universal health coverage" advocated by WHO. In addition, the geographical environment in western China is relatively complex, for instant, Xinxiang, Inner Mongolia, Tibet, and other provinces (autonomous regions and municipalities) geographic area is larger, high altitude, low population density, Guangxi, Guizhou, Chongqing and other provinces (autonomous regions and municipalities) are mostly mountainous, traffic inconvenience. These unique climatic and geographical conditions also affect the geographical equity of health resource allocation in western China. The equity of geographical distribution of health resources will affect the accessibility of health resources, thus affecting the health equity of residents[53]. Thus, the government must improve the accessibility of health resources through tiered medical services, medical treatment alliances, strong economic investments, Internet hospitals and other diversity methods [54-56]. Moreover, the government can also use Geographic Information System (GIS) to further improve the accessibility of health resources distribution.

Thirdly, the inequity of health resources distribution was mainly came from the intra-group. Based on the analysis of the Theil index contribution rate in the two dimensions, the intra-group contribution rate was all over $60 \%$, higher than the inter-group. This result indicate that the inequality of resource allocation is mainly caused by the unequal distribution of health resources within the provinces (autonomous regions and municipalities) in western China. This may be caused by China's current resource allocation policies, the different population densities in different cities can be one of the reasons. The unique geographical feature of western China which described above is further exacerbated the situation. Some western rural areas are extremely inconvenient transportation, vehicles cannot reach, have caused inconvenience to the construction and transportation health resources. Moreover, the health resources allocation is related to economic development[57], level of economic development amongst cities and counties can lead to the unequal distribution of health resources inside the provinces (autonomous regions and municipalities). Therefore, when formulating regional development plans, the government should consider the economic strength of different regions and make targeted bias policies to reduce the inequity in the human resources allocation caused by economic reason.

Fourthly, we found that human resources distributive inequality was more obvious in the intra-group in the two dimensions. In 2018 , the intra-group contribution rate of human resources in the population dimension has reached over $95 \%$. Moreover, the intra group distributive inequality of medical (assistant) physicians showed an increasing trend in population dimension. This phenomenon can be owned to the relevant policies and the characteristics of western China. From the perspective of relevant policies, the incentive systems such as salary and promotion for medical staff employed in the community medical institutions is insufficient. In addition, the attraction of relatively developed cities and large medical institutions leads to the low willingness of medical staff to work in primary medical institutions, which resulting in the differences of health resources distribution in urban and rural [58,59]. In terms of the western own characteristics, Yunnan, Tibet, and other places with high altitude, easy to hypoxia, it is difficult for staff to adapt. In addition, there are many ethnic minority areas in western China, such as Ningxia, Guangxi, Guizhou etc. These areas have their own traditional culture, and most of the primary medical institutions are located in rural areas, which are more influenced by traditional culture and have more backward economic conditions. The factors above resulting in more difficult working environment, and staff are difficult to integrate into them, and it is difficult for them to obtain a sense of identity and sense of belonging. These factors restrict the introduction of professional talents, and the inequity human resources allocation in the western provinces (autonomous regions, municipalities). Human resource is one of the key resources of health resources[60]. Hence, the government should strengthen the training of medical personnel, moreover, we suggest that the training environment should be optimised by strengthening the incentive system and broadening the promotion channels to encourage more medical personnel to work in primary medical institutions, pay attention to the humanistic care for medical staff and help them integrate into local life to further realize health equity and improve the equity of human resources allocation.

The present study holds some limitations. Based on the "China 13th Five-Year Health and wellness Plan", the indexes of medical and health service system also include the number of general practitioners per 10,000 population and the proportion of beds in socially run hospitals in total number of hospital beds. Meanwhile, efficiency of health resource use is also a key factor affecting health equity and the capacity of health institutions to provide services[61]. However, due to the limited availability and integrity of data collection, the above problems were not further analysed in this paper.

\section{Conclusion}

Based on the research above, we found that in recent years, the total amount of health resources in western China has increased as a whole, and the government's measures to improve resource equity have played a role. However, the inequity of health resource distribution persists in western China. The 
major differences of health resources allocation existed in the geographical dimension and the intra-group, which may impact health resources accessibility. Moreover, the equity of human resources allocation was relatively poor, which warrants urgent promotion. These problems are also the key factors related to people's health. Hence, the stakeholders, including the government, health institutions and so on should pay more attention in solving problems to improve the equity of health resources allocation.

\section{Abbreviations}

GIS Geographic Information System

\section{Declarations}

\section{Availability of data and materials}

The data in this paper obtained from China statistical yearbook from 2015 to 2019, and the public data which released by the National Health and Family Planning Commission from 2015 to 2019

\section{Ethics approval and consent to participate}

The authors confirm that the ethical policies of the journal, as noted on the journal's author guidelines page, have been adhered to and the appropriate ethical review committee approval has been received.

\section{Consent for publication}

Not applicable

\section{Competing interests}

The authors declare that they have no competing interests

\section{Funding}

Health and Health Policy Research Center of Guangxi Medical University; 2020 Project of Guangxi Young Teachers' Ability Improvement(2020KY43004)

\section{Contributions}

Zheng Wang, Haoyu He and Xi Liu: Prepared all the figures and table Wrote the main manuscript test, contributions were consistent. Qiming Feng and Bo Wei: methodology designed. All authors read and approved the final manuscript.

\section{Acknowledgements}

Not applicable.

\section{References}

1. Gao JM, Yang JJ. overview of health fair. Health Economics Research 2014, 51-54.

2. He MH, Yu L, He J, Wei Y. Study on the status and equty og health services resources allocation in China. The Chinese Health Serice Management. 2013;30:197-9.

3. Cao Y, Yan Y. A study on health resource allocation efficiency in JiangSu province from the perspective of health equity. Health Economics Research 2016, 18-21.

4. Wang S, Xu J, Jiang X, Li C, Li H, Song S, Huang E, Meng Q. Trends in health resource disparities in primary health care institutions in Liaoning Province in Northeast China. Int J Equity Health 2018, 17, doi:10.1186/s12939-018-0896-8.

5. Song XR, Zhu JL, Liu QK, Chen C, Zhang JY, Li YH. Ananlysis on the fairness of health resource allocation and utilization ad utilization in 31 provinces and cities in China. Chinese Journal of Social Medicine. 2020;37:545-8.

6. Liu T, Li JX, Chen J, Yang SL. Regional Differences and Influencing Factors of Allocation Efficiency of Rural Public Health Resources in China. Healthcare (Basel). 2020;3:270.

7. Wang S, Xu J, Jiang X, Li C, Li H, Song S, Huang E, Meng Q. Trends in health resource disparities in primary health care institutions in Liaoning Province in Northeast China. Int J Equity Health 2018, 17, doi:10.1186/s12939-018-0896-8. 
8. Xu K, Zhang K, Wang D, Zhou L. Trend in distribution of primary health care professionals in Jiangsu province of eastern China. Int J Equity Health. 2014;13:117. doi:10.1186/s12939-014-0117-z.

9. Ren Z, Song P, Chang X, Wang J, An L. Inequality of obstetric and gynaecological workforce distribution in China. Int J Equity Health 2018 , 17, doi:10.1186/s12939-017-0716-6.

10. Sun J, Luo H. Evaluation on equality and efficiency of health resources allocation and health services utilization in China. Int J Equity Health 2017 , 16 , doi:10.1186/s12939-017-0614-y.

11. Li F, Yuan QQ, Chen DD. Evaluation of Structure,Equity and Utilization Efficiency of Chinaï 14 \\s Health Resource Allocation. Medicine and Society $2020,33$.

12. Pan LM, Jiang YW, Wei LS, Chen JJ, Zhou B. Study on equity and efficiency of health resource allocation in China based on HRAD and DEA. Soft Science of Health. 2021;35:37-41.

13. Tang S, Meng Q, Chen L. Tackling the challenges to health equity in China. The Lancet. 2008;9648:1493-501.

14. National Bureau Statistics Of China. China Statistical Yearbook. http://www.stats.gov.cn/tjsj/ndsj/ (accessed on 2020-10-21).

15. Bureau Statstics Of Shaanxi. Shaanxi statistic yearbook. http://tjj.shaanxi.gov.cn/tjsj/ndsj/tjnj/ (accessed on 2020-12-21).

16. Bureau Statstics Of Sichuan. Sichuan statistic yearbook. http://tjj.sc.gov.cn/scstjj/c105855/nj.shtml (accessed on 2020-12-21).

17. Bureau Statstics Of Yunnan. Yunnan statistic yearbook. http://stats.yn.gov.cn/tjsj/tjnj/ (accessed on 2020-12-21).

18. Bureau Statstics Of Guizhou. Guizhou statistic yearbook. http://stjj.guizhou.gov.cn/tjsj_35719/sjcx_35720/gztjnj_40112/tjnj2018/(accessed on 2020-1221).

19. Bureau Statstics Of Guangxi. Guangxi statistic yearbook. http://tjj.gxzf.gov.cn/tjsj/tjnj/ (accessed on 2020-12-21).

20. Bureau Statstics Of Gansu. Gansu statistic yearbook. http://tjj.gansu.gov.cn/HdApp/HdBas/HdClsContentMain.asp?Classld=70 (accessed on 2020-1221).

21. Bureau Statstics Of Qinghai. Qinghai statistic yearbook. http://tj.qinghai.gov.cn/tjData/qhtjnj/ (accessed on 2020-12-21).

22. Bureau Statstics Of Ningxia. Ningxia statistic yearbook. http://nxdata.com.cn/publish.htm?cn=G01 (accessed on 2020-12-21).

23. Bureau Statstics Of Tibet. Tibet statistic yearbook. http://tjj.xizang.gov.cn/ (accessed on 2020-12-21).

24. Bureau Statstics Of Inner mongolia. Inner mongolia statistic yearbook. http://tj.nmg.gov.cn/ (accessed on 2020-12-21).

25. Bureau Statstics Of Xinjiang. Xinjiang statistic yearbook. http://tjj.xinjiang.gov.cn/tjj/zhhv/list_nj1.shtml (accessed on 2020-12-21).

26. Bureau Statstics Of Chongqing. Chongqing statistic yearbook. http://tjj.cq.gov.cn/zwgk_233/tjnj/ (accessed on 2020-12-21).

27. Sun W. Study on the Efficiency and Equity of Health Resource Allocation in primary medical and health institutions in Jilin Province. master Type, Jilin University; 2020.

28. WAGSTAFF A, PACI P, VAN DOORSLAER E. On the measurement of inequalities in health. Soc Sci Med. 1991;33:545-57.

29. Yan-hui Z, Xiao-ning H, Tao BO. Study on the Equity of Health Resources Allocation in Primary Health Care Institutions in Beijing. Chinese Health Economics. 2020;39:46-9. doi:10.7664/CHE20200712.

30. Liu C. Analysis on Equity of General Practitioners Allocation: Based on Gini Coefficient and Theil Index. Chinese Health Economics. 2017;36:49-52.

31. Nan Z, Xiaojie S, Cheng L, Xin W, Kun L Analyzing the equity of health resources allocation in China based on Theil Index. / Zhang, Nan,et al. / / The Chinese Health Service Management. Chinese Health Service Management 2014, 31, 88-91.

32. Peng R, Deng M, Qin X, Feng J, Wei X, Fan D, Feng Q, Li Z. Research on the current and equity of health resource in allocation in Guangxi. Health Policy Research in China. 2020;13:57-63.

33. TANG S, ZHANG R, SI Y. Measurement of the equality of the drug welfare induction level of Chinese patients with chronic diseases in Gansu, Sichuan, Hebei, and Zhejiang based on the bivariate Theil-T Index method. Frontiers in Public Health 2020.

34. Zhang N, Sun XJ, Li C. Analyzing the equity of health resources allocation in China based on Theil Index. The Chinese Health Service Management 2014, $88-91$.

35. Wen D, Zheng YT, Zhao B. Research on the equity and efficiency og Guangxi medical and health resources allocation. Modern Preventive Mediclne. 2021;48:867-71.

36. Ying M, Bin Z, Jinlin L, Pengpeng J, Jingxian W, Yichen L, Xiaoyang S. Analysis on the Equity of Human Resource Allocation for Health in Western China: Based on the Resource Homogenei囚. ty Assumption. Chinese Health Economy. 2015;34:31-4.

37. Xia F, Zhang R, Leng Y, Fan Y, Du X. Research on the Equity of Community Health Resources allocation in Jilin Province. Health Economics Research 2016, 15-18.

38. Lu M, Ping T, Yukun X. Analysis on the allocation efficiency of medical and health resources in 12 western provinces: Based on DEA-Malmquist index analysis. Assets and Finances In Administration and Institution 2019, 41-42, doi:10.3969/j.issn.1674-585X.2019.08.021.

39. The State Concil. The 13th Five-Year Plan on Health and Wellness. (accessed on 2021-07-27).

40. National Bureau Statistics Of China. China Statistical Yearbook 2019. http://www.stats.gov.cn/tjsj/ndsj/2019/indexch.htm (accessed on 2020-10-21)..

41. Zhejiang Provencial Bureau Of Statistics. 2019 Zhejiang statistic yearbook. https://zjjcmspublic.oss-cn-hangzhou-zwynet-d01-

a.internet.cloud.zj.gov.cn/jcms_files/jcms1/web3077/site/flash/tjj/Reports1/2020\%E7\%BB\%9F\%E8\%AE\%A1\%E5\%B9\%B4\%E9\%89\%B420200929/2019\%E (accessed on 2021-1-12).

42. Shandong Provencial Bureau Of Statistics. 2019 Shandong statistic yearbook. http://tjj.shandong.gov.cn/tjnj/nj2019/indexch.htm (accessed on 2021-112). 
43. Hunan Provencial Bureau Of Statistics. 2019 Hunan statistic yearbook. http://222.240.193.190/19tjnj/indexch.htm (accessed on 2021-1-12).

44. Hubei Provencial Bureau Of Statistics. 2019 Hubei statistic yearbook. http://tjj.hubei.gov.cn/tjsj/sjkscx/tjnj/qstjnj/ (accessed on 2021-1-12).

45. Wenqing J. The Impact of Imbalanced Health Resource Allocation on the Residents'Health: Based on the Urban-Rural and Re囚. gional Perspective. Health resources allocation $35,55-57$.

46. Pang T, Wei B, Feng Q. Evaluation of health resource allocation in Guangxi in 2018 based on RSR. Soft Science of Health. 2020;34:80-4.

47. Sun J. Study on equity of health resource allocation in Guangxi. Rural economy science technology. 2017;28:255-8.

48. Hu W, Yang J, Xu R, Ni R, Meng X, Yu EE. Chinese General Practice. 2015;18:2628-31.

49. Chen S. Study on Evaluation on Community Health Service Resourse in China under the Background of Hierachial Medical System. master Type. Nanjing University of Chinese Medicine; 2017.

50. Zhao DJ, Wang DB, Xu XJ, Hu ZZ. The equity analysis of health personnel allocation of city in Anhui. Anhui Medical Journal. 2013;34:845-7.

51. Liu M, Xie DG, Li W, Cui XJ, Ma XE. Analysis on the fairness of health human resources allocation in community health service centers in China. Soft Science of Health. 2020;34:56-62.

52. Liang S. Research on the Core Competence Building of Basic Public Health Service Workers in Primary Health Care Sectors in Western China. Type D, The PLA Army Medical University, 2020.

53. Le Y, Hong-man W. Analysis on the Allocation Equality in Health Service Resources in Beijing. Chinese Health Economics. 2018;37:37-40. doi:10.7664/CHE20181110.

54. Yan Z, Shiqing L, Xuejiao L, Liang Z. Analysis on the Demand and Evolution of Health Services for Residents in the New Period. Chinese Health Economics. 2020;39:5-7. doi:10.7664/CHE20200501.

55. Chunyan X, Aijie Y. Research on Equity of Medical and Health Resources Allocation in Shandong Province. Medicine Society. 2018;31:18-21.

56. Fan D, Qin X, Deng M, Wei X, Feng Q. Research on the Situation and Equity of Health Resource Allocation in Guangxi in 2015-2018. China's Health Economy. 2020;39:50-4.

57. Shao W, Li J, Ma T, Zhang L, Zhang Q, Li X, Ma J. Analysis on Status Quo and Equity of Medical and Health Resources Allocation in Jilin Province. Medical Society. 2021;34:49-53.

58. Huangshang Y, Deng M, Qin X, Feng Q. Study on the Allocation and Equity of Nursing Human Resources in Guangxi during the "13th Five-year Plan" Period. Health Economics Research 38, 58-61.

59. Jing L, Xianming H. Equity analysis of health resources allocation in Shanxi Province. Chinese Journal of Health Policy. 2021;14:73-8.

60. Su B, Liu S, Lu Y, Yao F, Zhao Y, Zheng X. Evaluation of human resource allocation of primary healthcare in China: Based on agglomeration degree. Chinese Journal of Health Policy. 2021;14:49-54.

61. Li Q, Wei J, Jiang F, Zhou G, Jiang R, Chen M, Zhang X, Hu W. Equity and efficiency of health care resource allocation in Jiangsu Province, China. Int J Equity Health 2020, 19, doi:10.1186/s12939-020-01320-2.

\section{Figures}

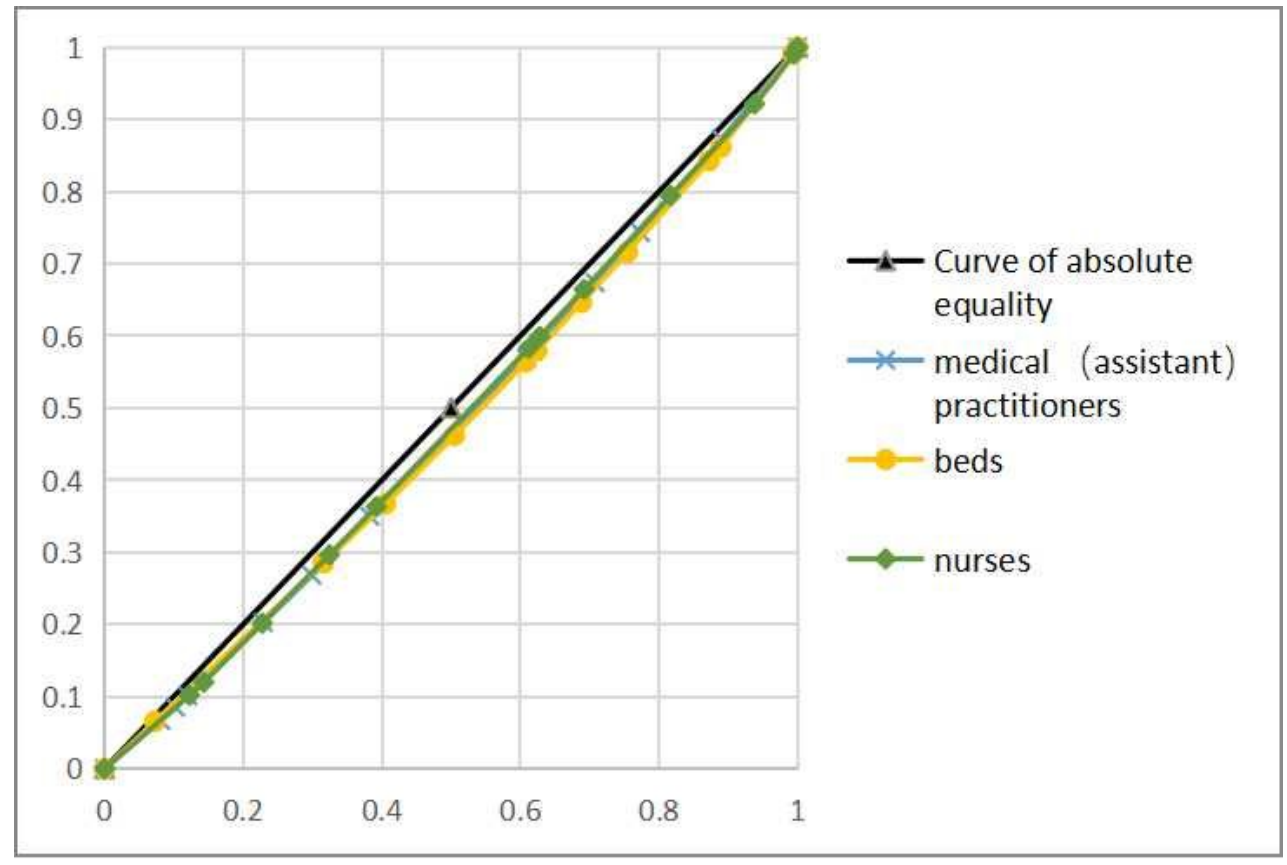

Figure 1

Lorentz curve of health resources distribution based on population dimension in western China from 2014 to 2018

Page $10 / 12$ 


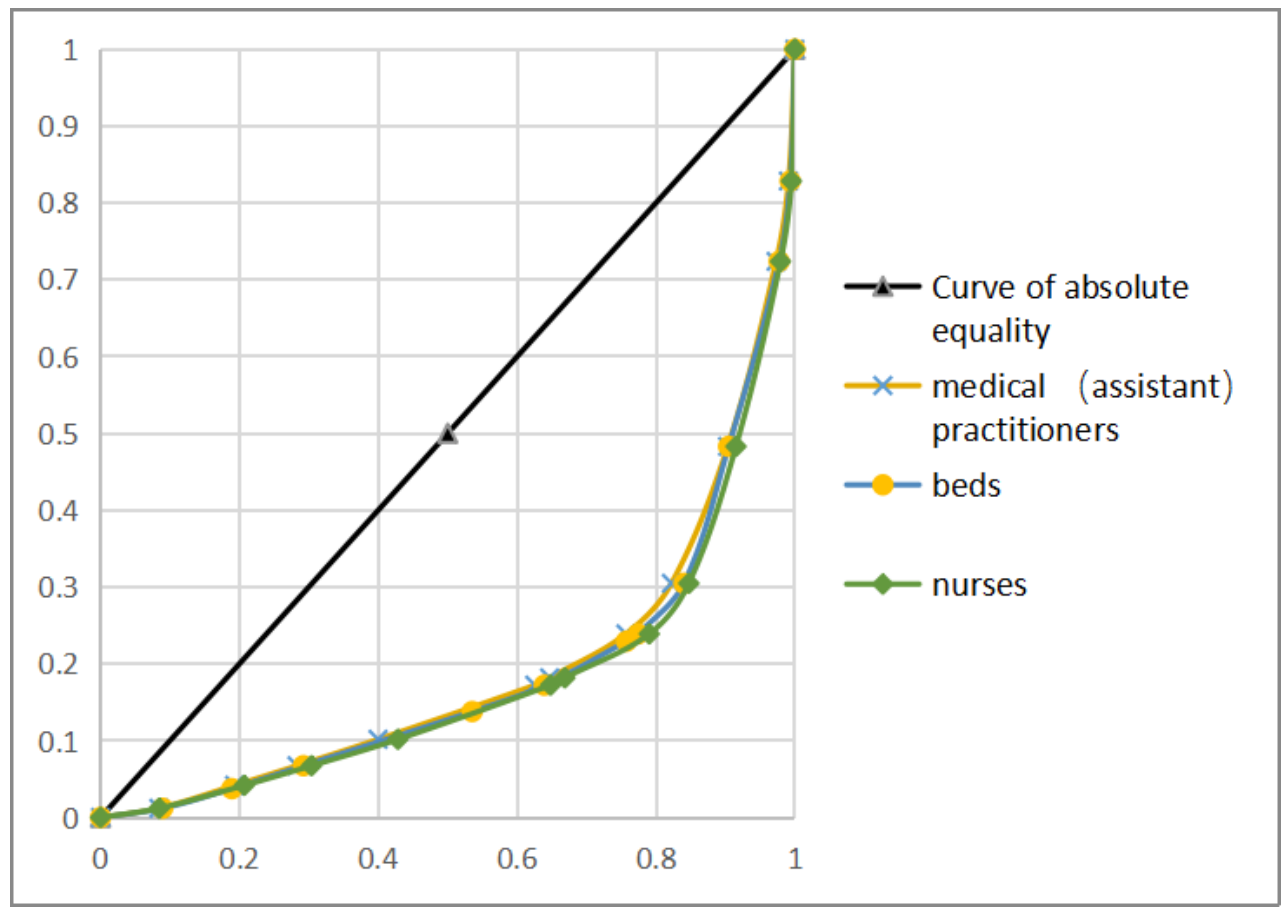

Figure 2

Lorentz curve of health resources distribution based on geographical dimension in western China from 2014 to 2018

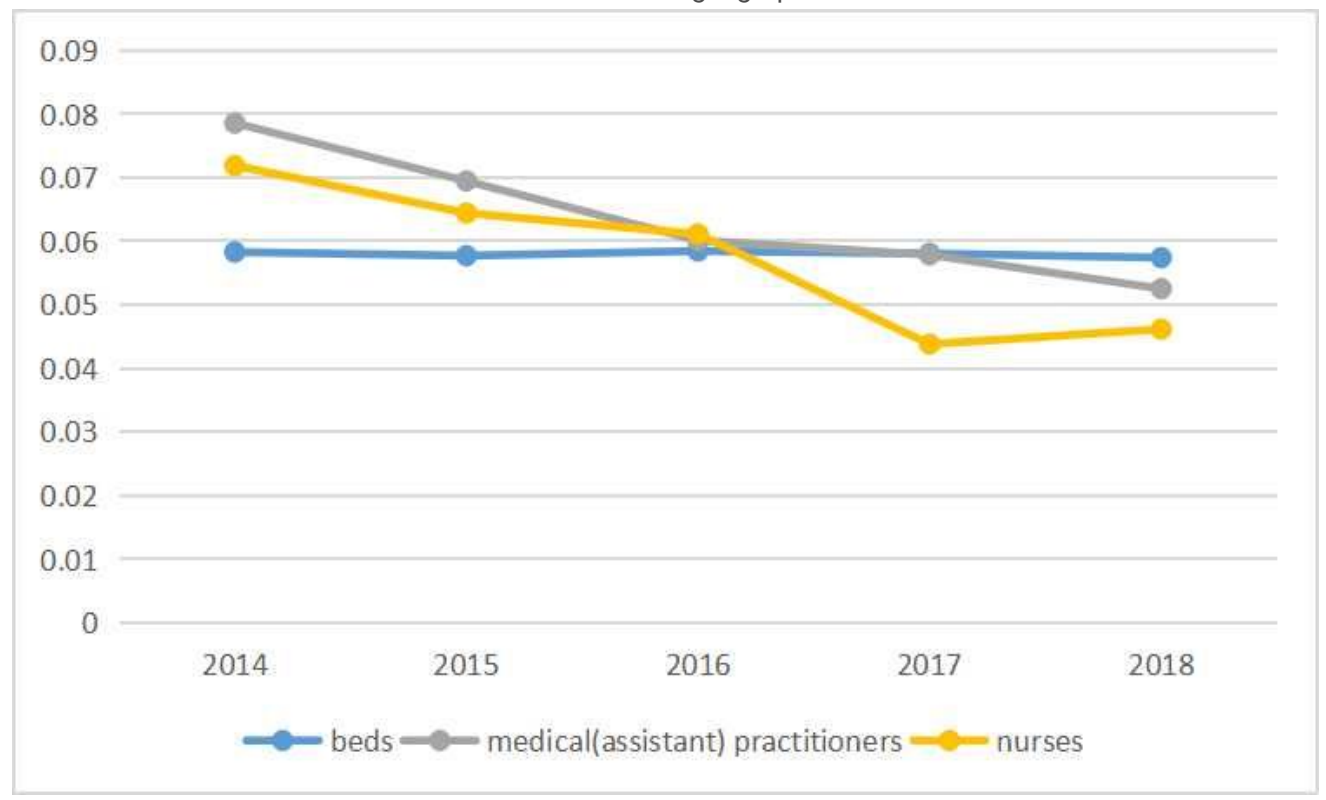

Figure 3

Gini coefficient variation tendency of health resources base on population dimension in western China from 2014 to 2018 


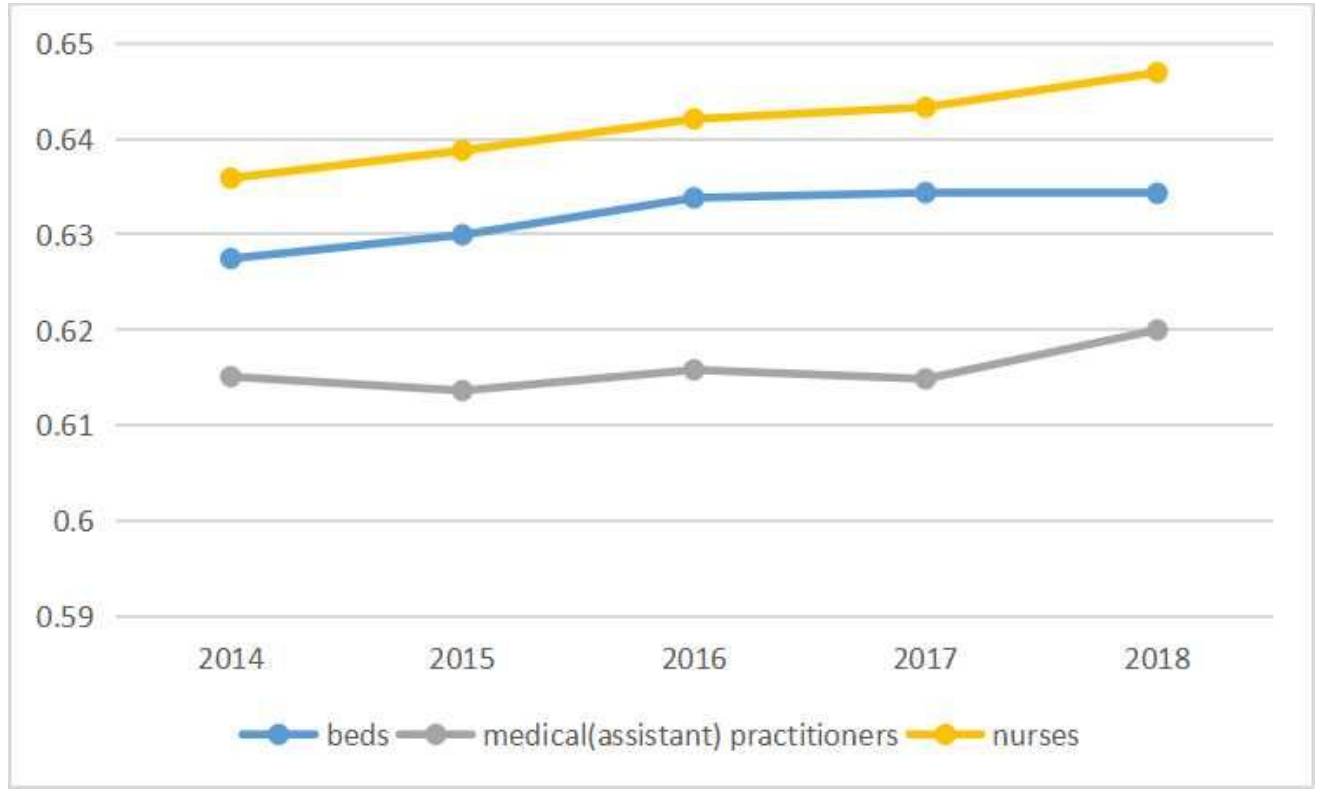

\section{Figure 4}

Gini coefficient variation tendency of health resources base on geographical dimension in western China from 2014 to 2018 